\title{
Corrigendum
}

\author{
June 2002
}

Volume 109 | Number 11

\section{GAD65-reactive $T$ cells are activated in patients with autoimmune type 1a diabetes}

Vissia Viglietta, Sally C. Kent, Tihamer Orban, and David A. Hafler

J. Clin. Invest. 109:895-903 (2002). DOI:10.1172/JCI200214114.

In the final stages of the production process, an error was introduced into figure 1 . The correct version of the figure and the legend appear below.

a

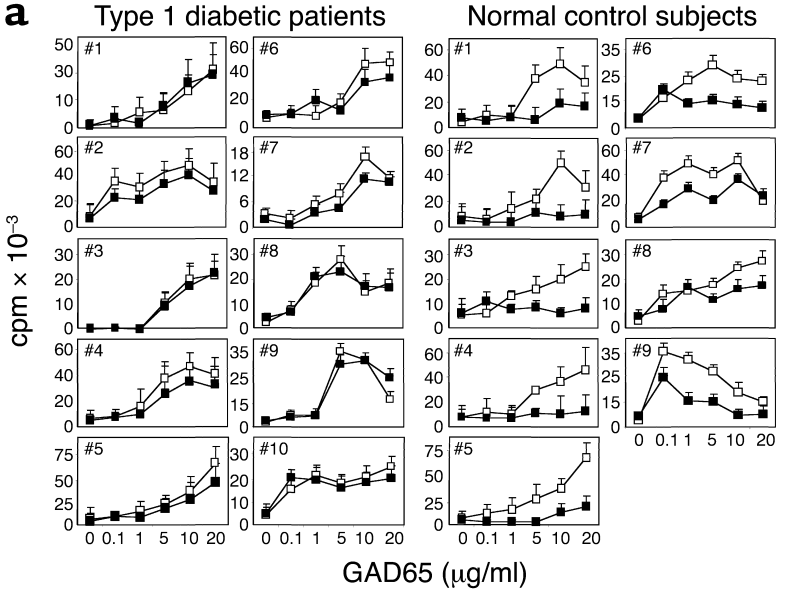

C
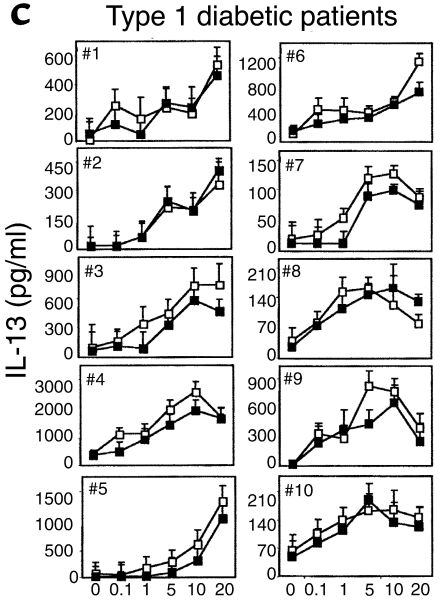

Normal control subjects

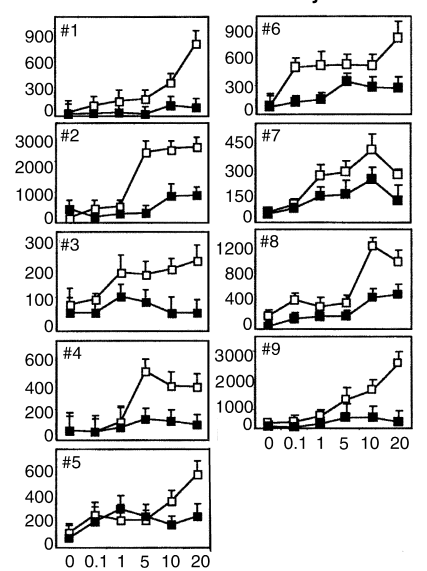

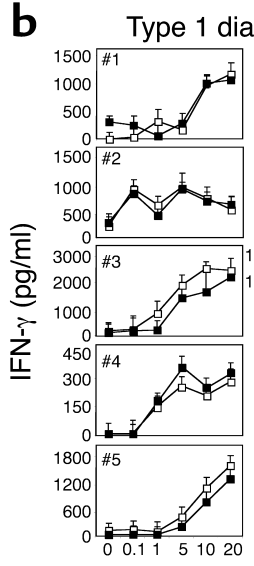

d
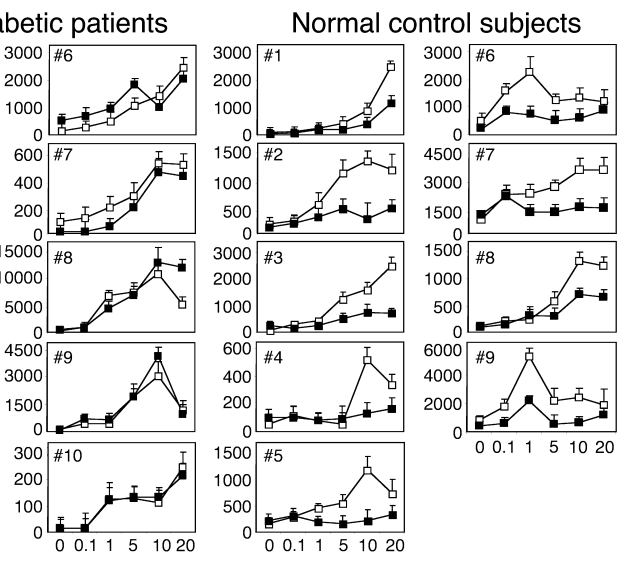

GAD65 ( $\mu \mathrm{g} / \mathrm{ml})$

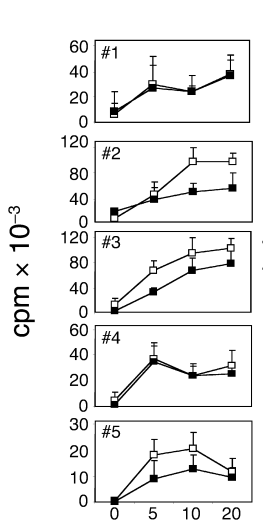

Long-term diabetic patients

IFN- $\gamma$

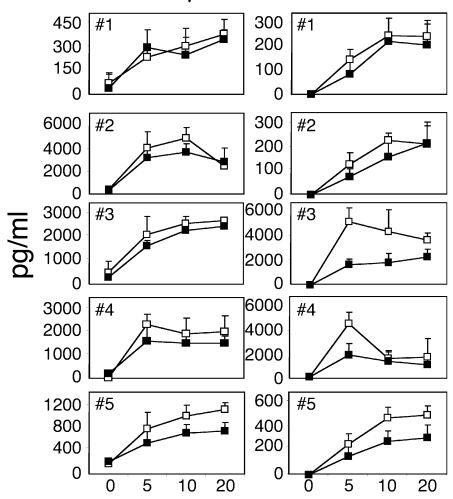

GAD65 $(\mu \mathrm{g} / \mathrm{ml})$

Figure 1

Response to GAD65 in individual type 1 diabetes patients and normal control subjects with blocking costimulatory signals. PBMCs from type 1 diabetes patients and normal control subjects were cultured with different concentrations of GAD65 $(0.1-20 \mu \mathrm{g} / \mathrm{ml}) \mathrm{or} T \mathrm{~T}(0.01-1 \mathrm{Lf} / \mathrm{ml})$ in the presence or absence of anti-CD28 F(ab') fragments. After 5 days of culture, rhlL-2 was added to each of ten replicates established for each antigen concentration with or without blocking Ab's. After an additional 5-7 days of culture, supernatants were collected for cytokine detection, and proliferation was assessed by $\left[{ }^{3} \mathrm{H}\right]$ thymidine incorporation. (a) T cell proliferation in 19 separate experiments. Each symbol represents the mean thymidine uptake of ten replicate cultures $\pm \mathrm{SE}$, stimulated only with the antigen (open squares) or with antigen plus anti-CD28 F(ab') fragment (filled squares). T cells from both patients and controls proliferated in response to GAD65, and blockade of CD28 costimulatory pathway inhibited GAD65 proliferation and (b and $\mathbf{c}$ ) cytokine secretion in healthy subjects but not in patients with type 1 diabetes. A summary of all the new-onset patients and controls is shown in Figure 2. (d) Proliferation and cytokine secretion in five long-term diabetic patients. 